\title{
A method to estimate the frequency of chromosomal rearrangements induced by CRISPR/Cas9 multiplexing in Drosophila.
}

\author{
William A. $\mathrm{Ng}^{*}$ and Bruce H. Reed ${ }^{*}$
}

*Department of Biology, University of Waterloo, Waterloo, ON, Canada N2L 3G1.

I Author responsible for correspondence with journal:

Bruce H. Reed, Department of Biology, University of Waterloo, 200 University Avenue West, Waterloo, Ontario, Canada N2L 3G1. Phone: 519-888-4567 x38085, Fax: 519-746-0614

Email: reed@uwaterloo.ca

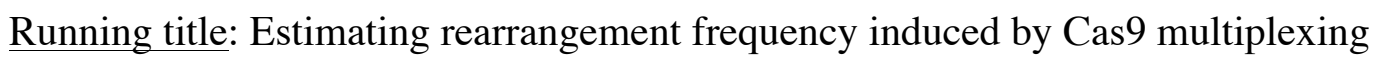

Key words: CRISPR/Cas9, multiplexing, chromosomal rearrangements 


\section{Abstract}

Using CRISPR/Cas9 to simultaneously induce mutations in two or more target genes, commonly referred to as multiplexing, may result in chromosomal rearrangements such as inversions or translocations. While this may be undesirable in some contexts, the ability to recover chromosomal rearrangements targeted to specific sites in the genome is potentially a powerful tool. Before developing such tools, however, it is first important to measure the frequency with which chromosome rearrangements are induced by CRISPR/Cas9 multiplexing. To do this, we have developed a selfselecting screening system using a Drosophila line that carries an autosomal pericentric inversion in what is known as the autosynaptic form. All progeny of normal females crossed to males of this autosynaptic stock are lethal due to excessive aneuploidy. If an inversion is induced within the female germline, and if it is analogous to the inversion in the male autosynaptic line, then it is possible to recover progeny in which aneuploidy is reduced and viability is restored. Using this selfselection method, we screened 130 females and recovered one new autosynaptic element. Salivary gland polytene chromosome analysis, PCR, and sequencing confirmed the recovery of a breakpoint induced precisely between the two sgRNA target sites. Overall, we demonstrate that CRISPR/Cas9 multiplexing can induce chromosomal rearrangements in Drosophila. Also, in using this particular system, the recovery of chromosomal rearrangements was not a high frequency event. 


\section{Introduction}

The development of CRISPR/Cas9 genome editing has been a game-changing advance in the field of genetics. Co-expression of Cas9 with an engineered single guide RNA (sgRNA) forms a sequence homology-dependent endonuclease that creates DNA double-stranded breaks (DSBs) within a target sequence (1). DSBs induced by Cas9 are repaired by endogenous DNA repair machinery, either through error-prone non-homologous endjoining (NHEJ), or through homology directed repair (HDR). The CRISPR/Cas9 system is routinely used to facilitate gene knockouts and gene replacements in most model genetic organisms, including Drosophila (2-4).

At the heart of CRISPR/Cas9 is the localization of a protein/RNA complex to a specific sequence, as specified by a 17-20 nucleotide region of the sgRNA molecule. Examples of using CRISPR/Cas9 to engineer chromosome rearrangements in other model systems include the creation of an oncogenic translocation in mice (5), translocations in yeast (6), translocations and inversions in C. elegans $(7,8)$, and inversions in zebrafish (9). This study represents the first report of a chromosomal rearrangement induced by CRISPR/Cas9 in the Drosophila germline.

Site specific chromosomal rearrangements in Drosophila have been recovered using FLP/FRT mediated recombination (10). This technology was also used to generate a collection of chromosomal deletions with molecularly defined breakpoints, known as the DrosDel collection (11). Each of these cases, however, depended on random integration of a $P$ element transposon carrying an FRT site. Subsequently, the design and recovery of deletions or rearrangements required knowing the genomic 
position and orientation of the insertions. The use of CRISPR/Cas9 multiplexing to generate chromosomal rearrangements would represent an improvement in that it can be used to target specific sequences and eliminates the need to map and characterize randomly generated insertions.

We wished to determine if CRISPR/Cas9 mutagenesis can be used to recover site specific chromosome rearrangements in Drosophila. To do this we developed a screening system based on autosynaptic chromosome elements (Fig.1). Due to the absence of meiotic recombination in Drosophila males, pericentric inversions, which are normally found in what is called their heterosynaptic form $(\operatorname{In}(2 L R) /+$, for example), can be maintained in what is known as the autosynaptic form (which is described as $L S(2) / / D S(2))(12-14)$.

We first recovered the autosynaptic form of a pericentric inversion with breakpoints at the base of $2 L$ and distal $2 R$. Crossing schemes were then designed to recover females that have Cas9 germline expression and ubiquitous expression of two sgRNAs targeted to the approximate positions of the pre-existing $\operatorname{In}(2 L R)$ breakpoints . These females were then crossed to males of the autosynaptic stock. In a selfselecting screen, where most progeny are lethal due to excessive aneuploidy, the only progeny to successfully complete development are expected to carry new autosynaptic elements derived from the female germline. From 130 females, we recovered one new autosynaptic element. Polytene chromosome analysis, PCR, and direct PCR sequencing of the PCR product of the anticipated $39 E ; 60 C$ breakpoint were all consistent with the inversion breakpoint being the product of CRISPR/Cas9 mutagenesis. Although we lack a definitive frequency for this event, we conclude that 
it is possible to recover chromosomal rearrangements via CRISPR/Cas9 mutagenesis of two target sequences. To the best of out knowledge, this represents the first documentation of a chromosomal rearrangement recovered by targeted CRISPR/Cas9 mediated mutagenesis in Drosophila.

\section{Results}

Many stocks carrying autosynaptic chromosome elements (Fig. 1) were recovered throughout the 1980's; at the time, they were considered useful for synthesis of duplications and deficiencies $(13,14)$. The development of FLP/FRT based methods for recovering deletions and rearrangements was a vast improvement over methods based on autosynaptic chromosome elements. Being obsolete as a technology, all autosynaptic stocks were discarded from the main stock collection centers (Bloomington Drosophila Stock Center and Kyoto Stock Center). We were able to recover a stock of $\operatorname{In}(2 L R) l t^{G 16[L]} b w^{\nu 32 g[R]}$ in the autosynaptic form using the product mimic method (12) (Fig. 2). In(2LR)lt $t^{G 16[L]} b w^{v 32 g[R]}$ has breakpoints in 2 Lhet;60E and 2 Rhet;59E and is a synthetic duplication of $59 E-60 E$; it was originally made using autosynaptic chromosome elements (15). Reciprocal crosses (females but not males can be outcrossed to lines with cytologically normal chromosomes) and polytene chromosome analysis were used to confirm that the stock was a bona fide $L S(2) / / D S(2)$ autosynaptic form of the $\operatorname{In}(2 L R)$ (Fig. 3).

Knowing the cytological breakpoints of $\operatorname{In}(2 L R) l t^{G l 6[L]} b w^{v 32 g[R]}$, we searched the Bloomington Drosophila Stock Center for one sgRNA line with a target slightly distal to 2 Lhet and a second sgRNA line having a target between $59 E$ and $60 E$. These target 
sites were chosen such that any combination of $L S(2) l t^{G 16} / / D S(2) * n e w^{*}$ or $L S(2)^{*} n e w^{*} / / D S(2) b w^{v 32}$ would be slightly hyperploid would also complement any mutation induced at the breakpoint target site. We chose TOE.GS00435, which targets the region upstream of CG2201 (map position 39E3) and TKO.GSO0793, which targets $c N-I I I B$ (map position 60C2).

A series of crosses culminated in the recovery of virgin females carrying nosCas9, which expresses $\operatorname{Cas} 9$ in the germline, together with TOE.GSO0435 and TKO.GS00793, which ubiquitously express the sgRNAs targeted to 39E3 and 60C2, respectively (see Materials and Methods and Fig. 4 for description of crossing scheme). These females were mated in small groups (5-6 per vial) to autosynaptic males $L S(2) l t^{G 16} / / D S(2) b w^{v 32 g}$. As expected, most crosses were non-productive and produced no adult progeny. One vial, however, produced numerous progeny and virgin females isolated from this culture were found to be fertile upon backcrossing to the autosynaptic stock - which is a confirmation that they carry autosynaptic chromosome elements complementary to $L S(2) l t^{G 16}$ and $D S(2) b w^{v 32 g}$. The segregation of visible markers allowed us to determine that these females carried a new $D S(2)$ autosynaptic chromosome element, which we call DS(2)CIA-1 (for CRISPR induced autosynaptic). None of the progeny, however, were consistent with recovery of a new $L S(2)$ element. Polytene squash analysis of $L S(2) l t^{G l 6} / / D S(2) C I A-1$ clearly revealed a breakpoint between 39E3 and 60C2 (Fig. 5). PCR primers designed to amplify regions surrounding the sgRNA target sequences of TOE.GSO0435 and TKO.GSO0793 were tested in different combinations using template DNA from control or $L S(2) l t^{G 16} / / D S(2) C I A-1$. As expected, forward and reverse primers for each target 
sequence generated product. Strikingly, we found a PCR product was generated using the forward primer for the TOE.GSO0435 target sequence with the reverse primer for the TKO.GSO0793 target sequence on template DNA from $L S(2) l t^{G 16} / / D S(2) C I A-1$. Control DNA (from $L S(2) l t^{G l 6} / / D S(2) b w^{v 32 g}$ ) did not generate a PCR product, and these results are consistent with a chromosomal breakpoint from one sgRNA target sequence to the other. This was confirmed by direct PCR sequencing (Fig. 6).

\section{Discussion}

We have reestablished autosynaptic stocks in order to develop a method for determining if CRISPR/Cas9 multiplexing can lead to chromosome rearrangements, and to measure the frequency of such events. In what was essentially a small-scale pilot screen, we targeted two regions of chromosome 2 by Cas 9 mutagenesis within the female germline. The germline of such females could potentially harbor a pericentric inversion $\operatorname{In}(2 L R)$ with breakpoints at $39 E 3$ and $60 C 2$. The screening method to recover the pericentric inversion is self-selecting. This is because a cross of females with cytologically normal chromosomes mated to males carrying an analogous pericentric inversion in the autosynaptic form is non-productive; all progeny of such a cross are non-viable due to excessive eneuploidy. If, however, the germline of a female harbours a pericentric inversion as described, this female will segregate complementary autosynaptic chromosome elements following a single recombination event within the inversion loop. Our screening strategy was successful and we were able to recover a chromosome rearrangement induced by CRISPR/Cas9 mutagenesis. 
In theory, it should be possible to recover both the $L S$ and $D S$ elements from the newly induced pericentric inversion. In our case, although we were able to demonstrate, for the first time in Drosophila, a targeted chromosome rearrangement using CRISPR/Cas9, we only recovered the $D S(2)$ element. Similar observations have been made in self-selecting autosynaptic screens that were performed using Xray mutagenesis (13). The explanation is found in the preferential and nonMendelian inheritance of the smaller element during the segregation of asymmetric dyads in female Drosophila. In our case, a single recombination event within the pericentric inversion generates dyads that greatly differ in size (roughly an entire chromosome arm). Here the smaller $D S(2)$ element is likely preferentially inherited by the oocyte nucleus while the larger $L S(2)$ element is preferential passed to a polar body.

The screen as presented was performed on a small scale as we only tested 130 females. By repeating this screen we will be able to determine the frequency with which chromosomal rearrangements are recovered, at least in the context of our particular screen design. It is clear, however, that the recovery of progeny, and hence the induction of pericentric inversions within the female germline, was not a high frequency event.

The possibility of using CRISPR/Cas9 to design and generate chromosome rearrangements could be useful for studies regarding pairing dependent transcriptional regulation. Here, translocations or inversions could be targeted to precise sequences in order to test and map pairing dependent regulatory regions for a gene-of-interest. If the efficiency of CRISPR/Cas9 proves sufficiently high, or could be enhanced, then it 
may be also be possible to design schemes to recover chromosome rearrangements in other Drosophila, and perhaps non-Drosophila species. Overall, our self-selecting screen represents a platform in which the recovery of chromosome rearrangements induced by CRISPR/Cas9 can be rigorously tested and optimized.

\section{Materials and Methods}

Drosophila stocks: All Drosophila cultures were raised on standard medium at $25^{\circ} \mathrm{C}$ under a 12 hour light/dark cycle regime, unless otherwise indicated. The following stocks were obtained from The Bloomington Drosophila Stock Center:

\#36569: $D f(2 R) o r^{B R-6}$, cn bw sp / In(2LR)lt ${ }^{G 16[L]} b w^{\nu 32 g[R]}$

\#68051: ysc v sev ${ }^{21} ; P\{y[+t 7.7] v[+t 1.8]=$ TOE.GSO0435\}attP4O

\#77019: y sc v sev ${ }^{21} ; P\{y[+t 7.7] v[+t 1.8]=$ TKO.GSO0793\}attP4O

\#54591: y $M\{w[+m C]=n o s-C a s 9 . P\} Z H-2 A w^{*}$

The following stocks were obtained from The Kyoto Stock Center:

\#101863: y; C(2L)RM-SH1 / F(2R)VH2, bw

\#116105: C(2L)RM-P2, $d p^{o v l} / F(2 R) 1, c n c b w$

The following stock is maintained in the Reed Lab Stock Collection:

b cn px sp

\section{Recovery of the autosynaptic form of $\operatorname{In}(2 L R) l t^{G 16[L]} b w^{v 32 g[R]}:$ (Also see Fig. 2.)}

Females of $\operatorname{In}(2 L R) l t^{G 16[L]} b w^{v 32 g[R]} / b c n p x s p$ were recovered and crossed to males of y; $C(2 L) R M-S H 1 / F(2 R) V H 2, b w$. Male progeny that were $C(2 L) R M-S H 1$ /

$F(2 R) V H 2, b w / D S(2) b w^{\nu 32 g}$ were recovered and backcrossed to $\operatorname{In}(2 L R) l t^{G 16[L]} b w^{\nu 32 g[R]}$

/ b cn px sp. Progeny of this cross were established as individual lines and those that 
produced $b$ progeny were selected as the autosynaptic stock $L S(2) l t^{G 16}, b / /$

$D S(2) b w^{v 32 g}$. Females

of $\operatorname{In}(2 L R) l t^{G 16[L]} b w^{\nu 32 g[R]} /$ If were then crossed to $L S(2) l t^{G 16}, b / / D S(2) b w^{\nu 32 g}$ males, permitting recovery of $L S(2) l t^{G 16}, b / / D S(2) b w^{\nu 32 g}$, If .

Screen for new autosynaptic elements induced by CRISPR/Cas9: Males were recovered that were $w$ nos-Cas9.P w / Y; TOE.GS00435 / + and these were crossed to $F M 7 /+; C y O /+$ females. Virgin female progeny of this cross that were $F M 7 h, w / y$ nos-Cas9.P; CyO / TOE.GSO0435 were collected and crossed to males of y sc $v \operatorname{sev}^{21}$; TKO.GS00793. Virgin females of this cross that were y nos-Cas9.P / y sc v sev ${ }^{21}$; TOE.GS00435/ TKO.GS00793 were collected and crossed to $L S(2) l t^{G 16}, b$ // $D S(2) b w^{\nu 32 g}$, If males. Rare progeny were backcrossed to $L S(2) l t^{G 16}, b / / D S(2) b w^{\nu 32 g}$, If males. Viable progeny from this cross indicated the recovery of a new autosynaptic stock. Progeny that were $b I f^{+}$indicated a new $D S(2)$ element while $b^{+} I f$ progeny indicated recovery of a new $L S(2)$ element (Also see Fig. 4).

Polytene Chromosome Squashes: Temporary salivary gland polytene chromosome squashes were prepared using a $2 \%$ natural orcein stain (Gurr's 23282) that was prepared in equal parts $45 \%$ acetic and $45 \%$ lactic acid and aged for 29 years. Polytene chromosome breakpoints were mapped with reference to Lefevre's photographic maps (16).

Molecular characterization of $\boldsymbol{D S}(2) C I A-1$ : In order to molecularly characterize the DS(2)CIA-1 breakpoint we extracted genomic DNA from a pooled group of 20-30 isogenic male flies using gSYNC DNA Extraction Kits (Geneaid) according to supplier's instructions. We used $2.5 \mu \mathrm{L}$ of isolated genomic DNA in $25 \mu \mathrm{L}$ PCR 
bioRxiv preprint doi: https://doi.org/10.1101/815431; this version posted October 22, 2019. The copyright holder for this preprint (which was not certified by peer review) is the author/funder, who has granted bioRxiv a license to display the preprint in perpetuity. It is made available under aCC-BY-NC 4.0 International license.

reactions (2X FroggaMix; FroggaBio) utilizing primers flanking the guide target regions at $\sim 400$ bp upstream and downstream of the sgRNA target sequences. PCR products were purified using GenepHlow Gel/PCR Kits (Geneaid) then sent out for Sanger sequencing using both forward and reverse primers. 


\section{Acknowledgements}

Stocks obtained from the Bloomington Drosophila Resource Center (NIH

P40OD018537) were used in this study. This work was supported by a grant to

B.H.R. from the Natural Sciences and Engineering Research Council of Canada

(NSERC RGPIN-2015-04458).

\section{References}

1. Jinek M, Chylinski K, Fonfara I, Hauer M, Doudna JA, Charpentier E. A programmable dual-RNA-guided DNA endonuclease in adaptive bacterial immunity. Science. 2012;337(6096):816-21. doi: 10.1126/science.1225829. PubMed PMID: 22745249; PMCID: PMC6286148.

2. Bassett AR, Tibbit C, Ponting CP, Liu JL. Highly efficient targeted mutagenesis of Drosophila with the CRISPR/Cas9 system. Cell Rep. 2013;4(1):220-8. doi: 10.1016/j.celrep.2013.06.020. PubMed PMID: 23827738; PMCID: PMC3714591.

3. Gratz SJ, Cummings AM, Nguyen JN, Hamm DC, Donohue LK, Harrison MM, Wildonger J, O'Connor-Giles KM. Genome engineering of Drosophila with the CRISPR RNA-guided Cas9 nuclease. Genetics. 2013;194(4):1029-35. doi: 10.1534/genetics.113.152710. PubMed PMID: 23709638; PMCID: PMC3730909.

4. Ma D, Liu F. Genome Editing and Its Applications in Model Organisms. Genomics Proteomics Bioinformatics. 2015;13(6):336-44. doi: 10.1016/j.gpb.2015.12.001. PubMed PMID: 26762955; PMCID: PMC4747648.

5. Maddalo D, Manchado E, Concepcion CP, Bonetti C, Vidigal JA, Han YC, Ogrodowski P, Crippa A, Rekhtman N, de Stanchina E, Lowe SW, Ventura A. In vivo engineering of oncogenic chromosomal rearrangements with the CRISPR/Cas9 system. Nature. 2014;516(7531):423-7. doi: 10.1038/nature13902. PubMed PMID: 25337876; PMCID: PMC4270925.

6. Fleiss A, O'Donnell S, Fournier T, Lu W, Agier N, Delmas S, Schacherer J, Fischer G. Reshuffling yeast chromosomes with CRISPR/Cas9. PLoS Genet. 2019;15(8):e1008332. doi: 10.1371/journal.pgen.1008332. PubMed PMID: 31465441 ; PMCID: PMC6738639.

7. Iwata S, Yoshina S, Suehiro Y, Hori S, Mitani S. Engineering new balancer chromosomes in C. elegans via CRISPR/Cas9. Sci Rep. 2016;6:33840. doi: 10.1038/srep33840. PubMed PMID: 27650892; PMCID: PMC5030659.

8. Chen X, Li M, Feng X, Guang S. Targeted Chromosomal Translocations and Essential Gene Knockout Using CRISPR/Cas9 Technology in Caenorhabditis elegans. Genetics. 2015;201(4):1295-306. doi: 10.1534/genetics.115.181883. PubMed PMID: 26482793; PMCID: PMC4676527. 
9. Xiao A, Wang Z, Hu Y, Wu Y, Luo Z, Yang Z, Zu Y, Li W, Huang P, Tong X, Zhu Z, Lin S, Zhang B. Chromosomal deletions and inversions mediated by TALENs and CRISPR/Cas in zebrafish. Nucleic Acids Res. 2013;41(14):e141. doi: 10.1093/nar/gkt464. PubMed PMID: 23748566; PMCID: PMC3737551.

10. Golic KG, Golic MM. Engineering the Drosophila genome: chromosome rearrangements by design. Genetics. 1996;144(4):1693-711. PubMed PMID: 8978056; PMCID: PMC1207720.

11. Ryder E, Ashburner M, Bautista-Llacer R, Drummond J, Webster J, Johnson G, Morley T, Chan YS, Blows F, Coulson D, Reuter G, Baisch H, Apelt C, Kauk A, Rudolph T, Kube M, Klimm M, Nickel C, Szidonya J, Maroy P, Pal M, Rasmuson-Lestander A, Ekstrom K, Stocker H, Hugentobler C, Hafen E, Gubb D, Pflugfelder G, Dorner C, Mechler B, Schenkel H, Marhold J, Serras F, Corominas M, Punset A, Roote J, Russell S. The DrosDel deletion collection: a Drosophila genomewide chromosomal deficiency resource. Genetics. 2007;177(1):615-29. doi: 10.1534/genetics.107.076216. PubMed PMID: 17720900; PMCID: PMC2013729.

12. Craymer L. Techniques for manipulating chromosomal rearrangements and their application to Drosophila melanogaster. I. Pericentric inversions. Genetics. 1981;99(1):75-97. PubMed PMID: 6804304; PMCID: PMC1214493.

13. Gubb D, McGill S, Ashburner M. A selective screen to recover chromosomal deletions and duplications in Drosophila melanogaster. Genetics. 1988;119(2):377-90. PubMed PMID: 3135239; PMCID: PMC1203420.

14. Ashburner M, Golic KG, Hawley RS. Drosophila : a laboratory handbook. 2nd ed. Cold Spring Harbor, N.Y.: Cold Spring Harbor Laboratory Press; 2005. xxviii, 1409 p. p.

15. Gubb D, Roote J, Coulson D, Henchcliffe C, Lyttle T, Reed B. Autosynaptics made easy. Drosophila Information Newsletter 1991;2:10-3.

16. Lefevre G, Jr. A photographic representation and interpretation of the polytene chromosome of Drosophila melanogaster salivary glands. In: Ashburner M, Novitski E, editors. The Genetics and Biology of Drosophila melanogaster. London and New York: Academic Press; 1976. p. 31-66. 


\section{Figure 1.}

Autosynaptic Stocks: (A) Diagram showing the pairing arrangement of a normal chromosome paired with a pericentric inversion in the heterosynaptic form. Here the pericentric inversion occurs on a metacentric chromosome where one break is close to the centromere and the other is close to the telomere (upper diagram). Lower diagram shows an alternative way of depicting the same arrangement where pairing is only considered within the inversion loop. (B) Diagram showing the pairing arrangement of the same inversion but in the autosynaptic form (upper), and also showing an alternative way of depicting the same autosynaptic configuration (lower). From the lower diagrams it can be noted that autosynaptic stocks represent pericentric inversions where the two inversion breakpoints are no longer attached to a common centromere, but have been separated and are attached to the two homologous centromeres. (C) A Punnett square diagram showing the outcome of crossing a female that is heterozygous for a pericentric inversion crossed to a normal male. The heterozygous female can produce gametes that carry either the normal chromosome or the inversion, but can also produce gametes that carry the $L S$ or $D S$ autosynaptic chromosome elements as a result of a single exchange within the inversion loop. (D) A Punnett square diagram showing the outcome of crossing a female that is heterozygous for a pericentric inversion (or a female that carries the pericentric inversion in the autosynaptic form) crossed to a male carrying the inversion in the autosynaptic form. Females can produce gametes carrying the normal chromosome, the inversion, or either of the $L S$ or $D S$ autosynaptic chromosome elements. Due to the absence of crossing-over in male Drosophila, male gametes carry only the 
autosynaptic chromosome elements, and as a result the only viable progeny also carry the inversion in the autosynaptic form. (E) A Punnett square diagram showing the outcome of crossing a cytologically normal female to a male carrying the pericentic inversion it the autosynaptic form. In this example, progeny are $L S /+$ (upper diagram), which carry an extra copy of the chromosome's left arm and lack one copy of the distal region of the right arm, or $D S /+$ (lower diagram) which lack one copy of the left arm and carry an extra copy of the distal region of the right arm. Both genotypes are lethal due to excessive aneuploidy.

\section{Figure 2.}

\section{Recovery of the autosynaptic form of $\left.\operatorname{In}(2 L R) l t^{G 16[L}\right] b w^{\mathrm{v32g[R]}}$ via the product mimic}

method (12): (A) The first step in recovering the autosynaptic form of $\operatorname{In}(2 L R) l t^{G 16[L]} b w^{v 32 g[R]}$, which has breakpoints in 2 Lhet; $60 E$ and 2 Lhet; $59 E$ and also is associated with a duplication of the region 59E-60E, involves crossing females that are heterozygous for the inversion and a cytologically normal chromosome (here carrying the markers $b c n p x s p$ ) to males of that carry a compound $2 L$ chromosome with free $2 R$ chromosomes. The same females are crossed with the male progeny of the first cross, where the males carry the compound $2 L$ chromosome with one free $2 R$ chromosome and the $D S(2) b w^{v 32 g}$ autosynaptic chromosome element. The duplication of the distal $2 R$ region from the $59 E$ breakpoint to the telomere of $2 R$ represents a viable and tolerated degree of hyperploidy. Progeny of this cross are selected that carry the $L S$ and $D S$ elements. Males and females carrying $L S / / D S$ are crossed to each other to create the autosynaptic stock that will behave according to the Punnett square 
diagram as shown in Fig. 1D. (B) The $L S$ element as recovered is heterozygous for the visible mutation $b$, but $L S$ elements can become homozygous for $b$ following a 4 strand double exchange between the centromere and $b$ and can be selected within the $L S / / D S$ stock. (C) The original heterozygous inversion can be crossed to other visible markers, here shown is the dominant marker Irregular facets (If), and subsequently crossed to the $L S / / D S$ autosynaptic males to introduce markers onto the autosynaptic chromosome elements. Shown is the scheme used to recover $D S(2) b w^{v 32 g}$ carrying the visible dominant marker If.

\section{Figure 3.}

Cytological confirmation of the recovery of $L S(2) l t^{G 16} / / D S(2) b w^{v 32 g}:$ (A) Polytene chromosome squashes showing the inversion and chromosome breakpoints associated with $L S(2) l t^{G 16} / / D S(2) b w^{v 32 g}$. In this example, the telomeres of $2 R$ (arrows) are asynapsed and the unpaired telomere of $D S(2) b w^{v 32 g}$ is seen as separate and connected to the chromocenter. Most of $2 L$ ( $2 L$ telomeres are indicated by arrowheads) and the entire inversion loop region are synapsed. (B) Schematic diagram showing the arrangement of the pairing of the $L S(2) l t^{G 16}$ (red) and $D S(2) b w^{v 32 g}$ (blue) elements with the approximate positions of the breakpoints for each element indicated (black arrowheads).

\section{Figure 4.}

Autosynaptic chromosome elements can be used to design self-selecting screens:

(A) Outcome of a cross of females carrying cytologically normal chromosomes that 
were exposed to active Cas9 and sgRNAs targeted to $39 E 3$ and 60C2. In the event that no inversion was induced, all progeny are lethal due to excessive aneuploidy. (B) Outcome of the same cross in which a new inversion was induced between the two sgRNA target sequences. The only viable progeny will carry new $L S$ and $D S$ elements derived from single exchanges within the inversion loop of a female harbouring a pericentric inversion within her germline.

\section{Figure 5.}

Cytological confirmation of the recovery of $L S(2) l t^{G 16} / / D S(2) C I A-1$ : (A) Polytene chromosome squashes showing the inversion and chromosome breakpoints associated with $L S(2) l t^{G 16} / / D S(2) C I A-1$. In this example the homologous regions of the two elements have remained paired. (B) Schematic diagram showing the arrangement of the pairing of the $L S(2) l t^{G 16}$ (red) and $D S(2) C I A-1$ (blue) elements with the approximate positions of the breakpoints for each element indicated (black arrowheads). (C) Example of a polytene squash of $L S(2) l t^{G 16} / / D S(2) C I A$-1 where the $2 R$ telomeres are asynapsed. The base of $L S(2) l t^{G I 6}$ and the tip of $D S(2) C I A-1$ are indicated. The enlarged inset shows the $2 R$ telomere and the $39 E ; 60 C 2$ breakpoint associated with $D S(2) C I A-1$.

\section{Figure 6.}

Molecular characterization of $\boldsymbol{D S}(2) \boldsymbol{C I A}-1$ : (A) Overview of PCR strategy showing relative positions of primers designed to amplify the $60 C 2 \mathrm{sgRNA}$ target sequence of $G S 00793$ (primers $2+3$ ) and the 39E3;60C2 breakpoint using a primer 
flanking the proximal target sequence of sgRNA GS00435 (primers $1+3$ ). (B)

Results of PCR showing products for primers $2+3$ for in control

$\left(L S(2) l t^{G 16} / / D S(2) b w^{v 32 g}\right)$ and the new autosynaptic stock $\left(L S(2) l t^{G 16} / / D S(2) C I A-1\right)$ and

a product for primers $1+3$ only for the new autosynaptic stock and not the control.

\section{Figure 7.}

Sequence of $\boldsymbol{D S}(2) C I A-1$ breakpoint: (A) Reference sequence from region 39E3

showing the proximal sgRNA target sequence for TOE.GSO0435 (yellow highlight), the breakpoint position (arrow), and the region of sequence amplified using PCR

primers $1+3$ as show in Fig. 6 (blue underline). (B) Reverse complement of the reference sequence from region $60 C 2$ showing the sgRNA target sequence for TKO.GS00793 (yellow highlight), the breakpoint position (arrow), and the region of sequence amplified using primers $1+3$ as show in Fig. 6 (red underline). (C) Gas chromatogram DNA sequence readout generated by direct PCR sequencing of the primer $1+3$ product, sequenced using primer 3 . The molecular site of the $39 E 3 ; 60 C 2$ breakpoint of $D S(2) C I A-1$ is indicated (arrow), and sequence from 60C2 (overscored by red line) is shown as contiguous with sequence from $39 E 3$ (overscored by blue line). 


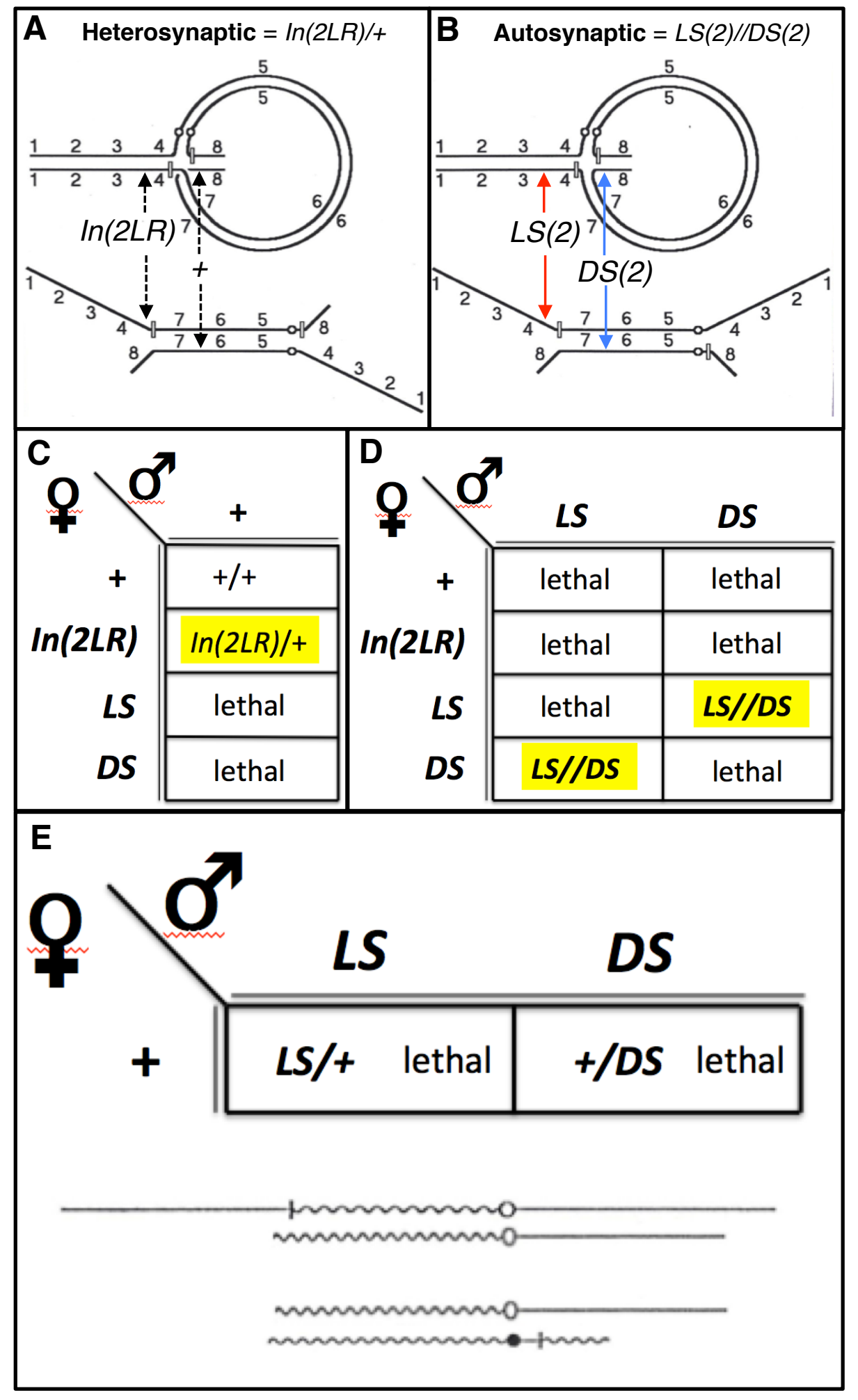

Figure 1 


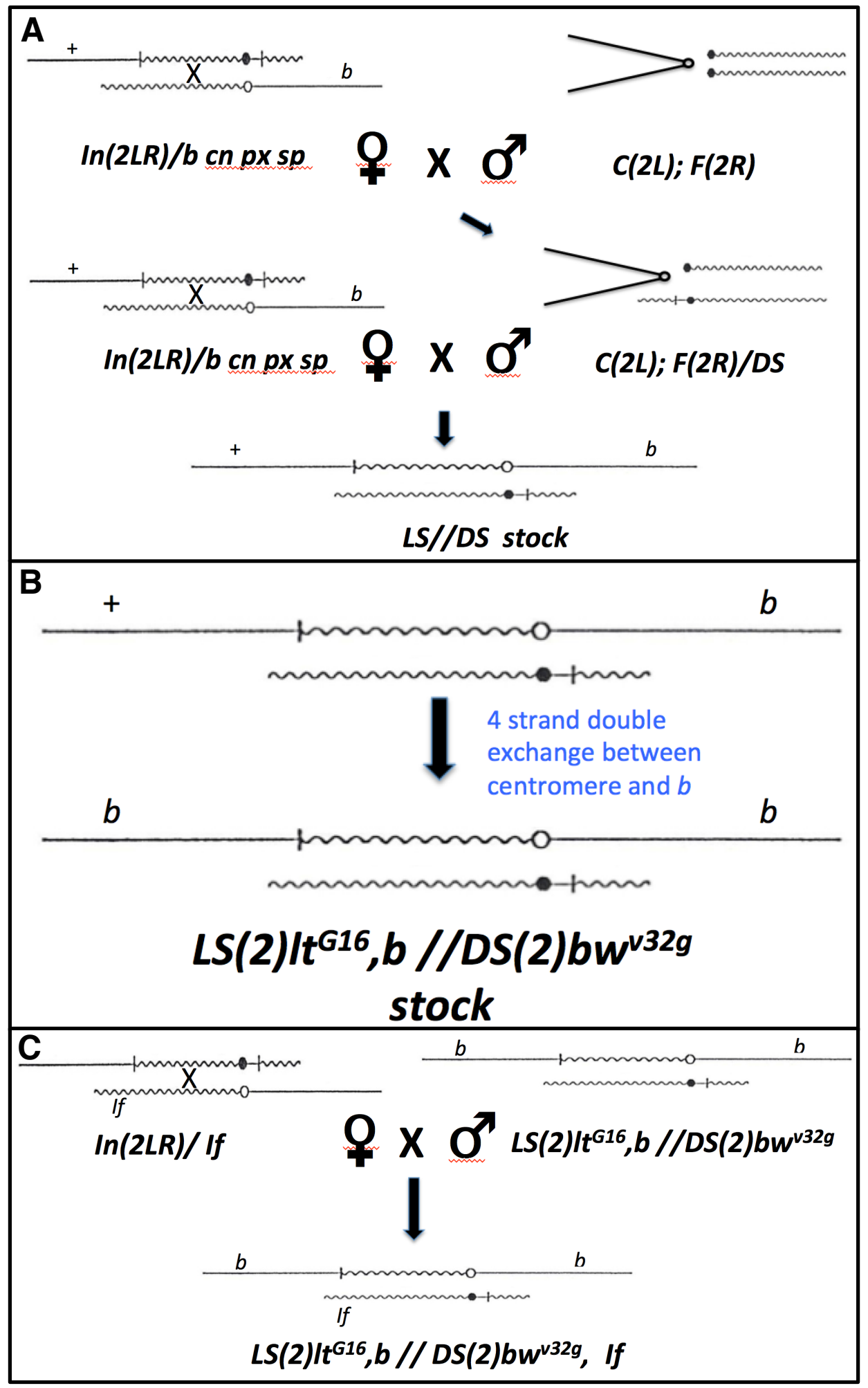

Figure 2 


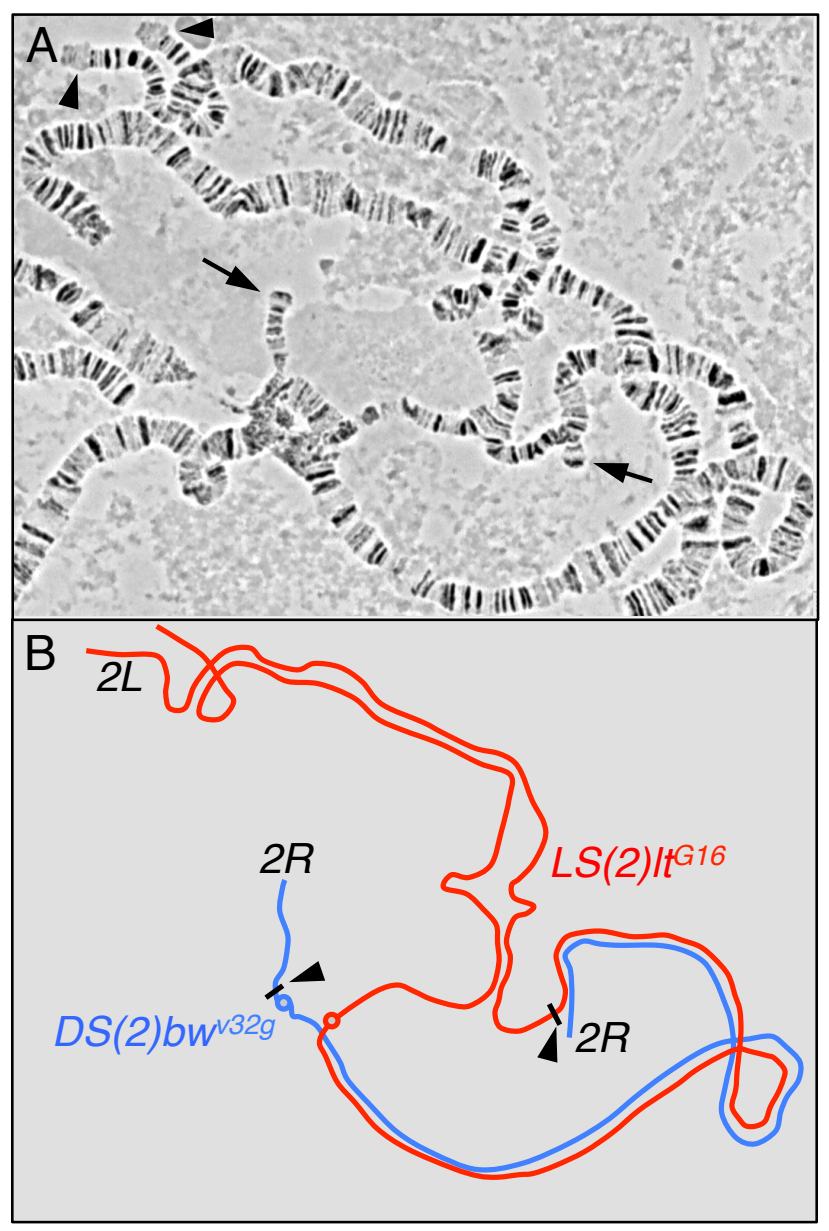

Figure 3 


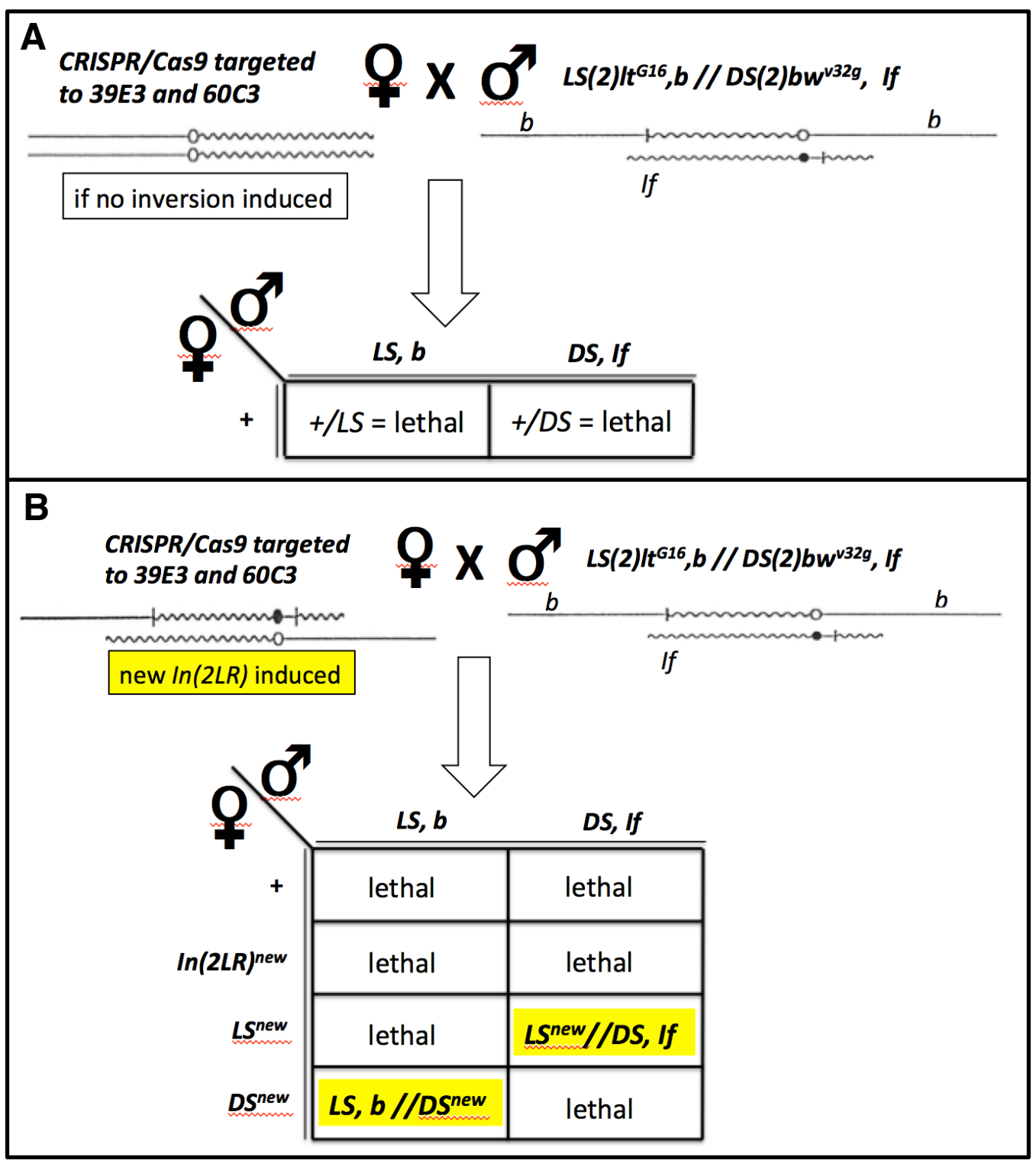

Figure 4 


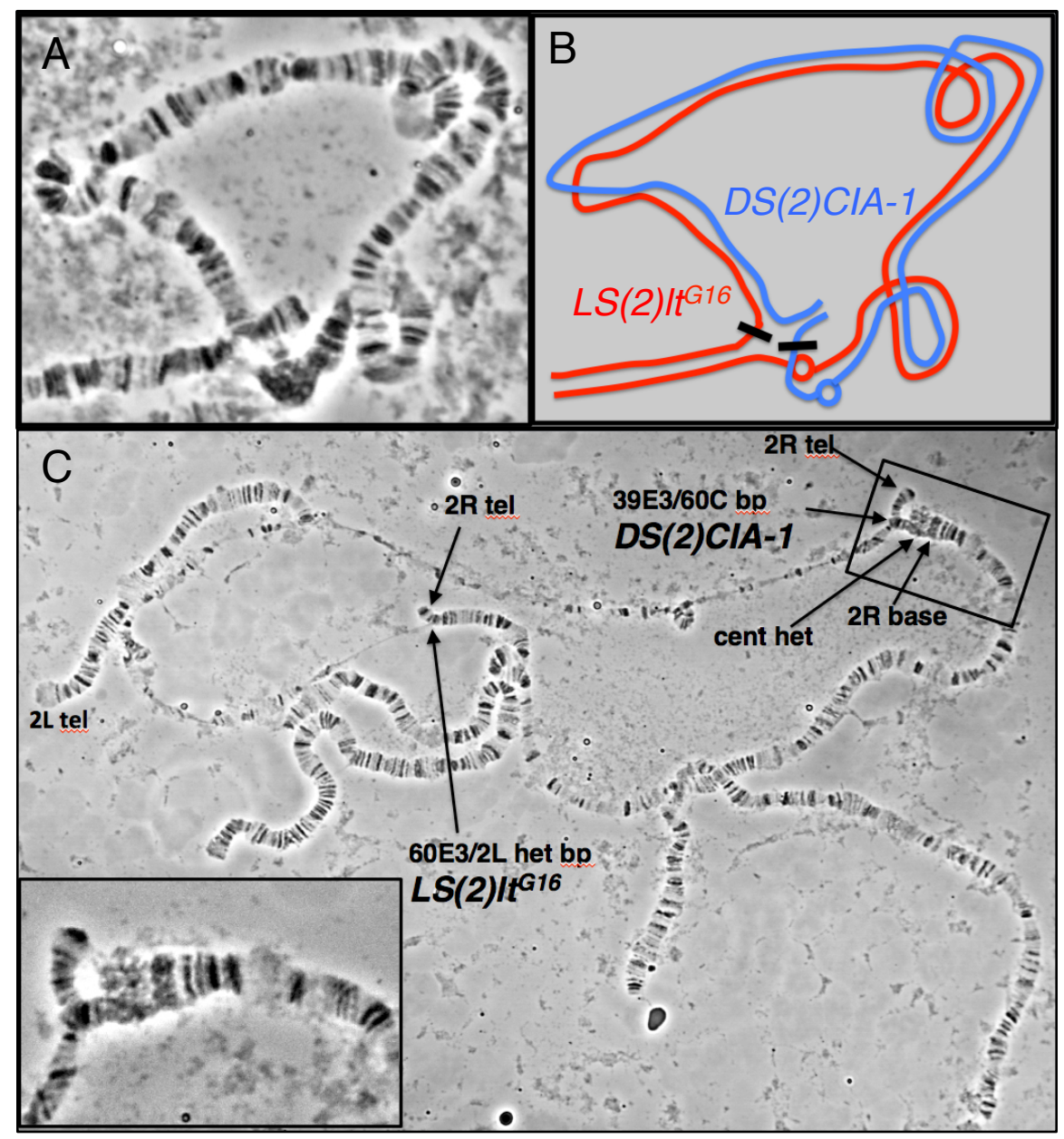

Figure 5 


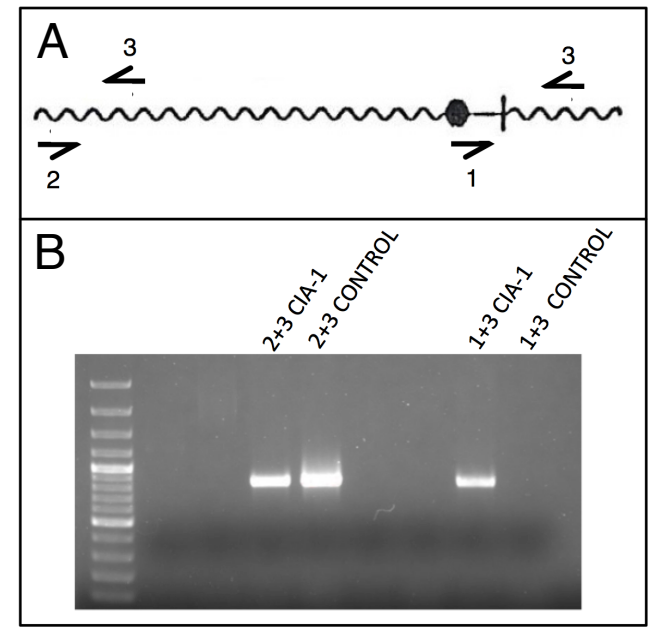

Figure 6 


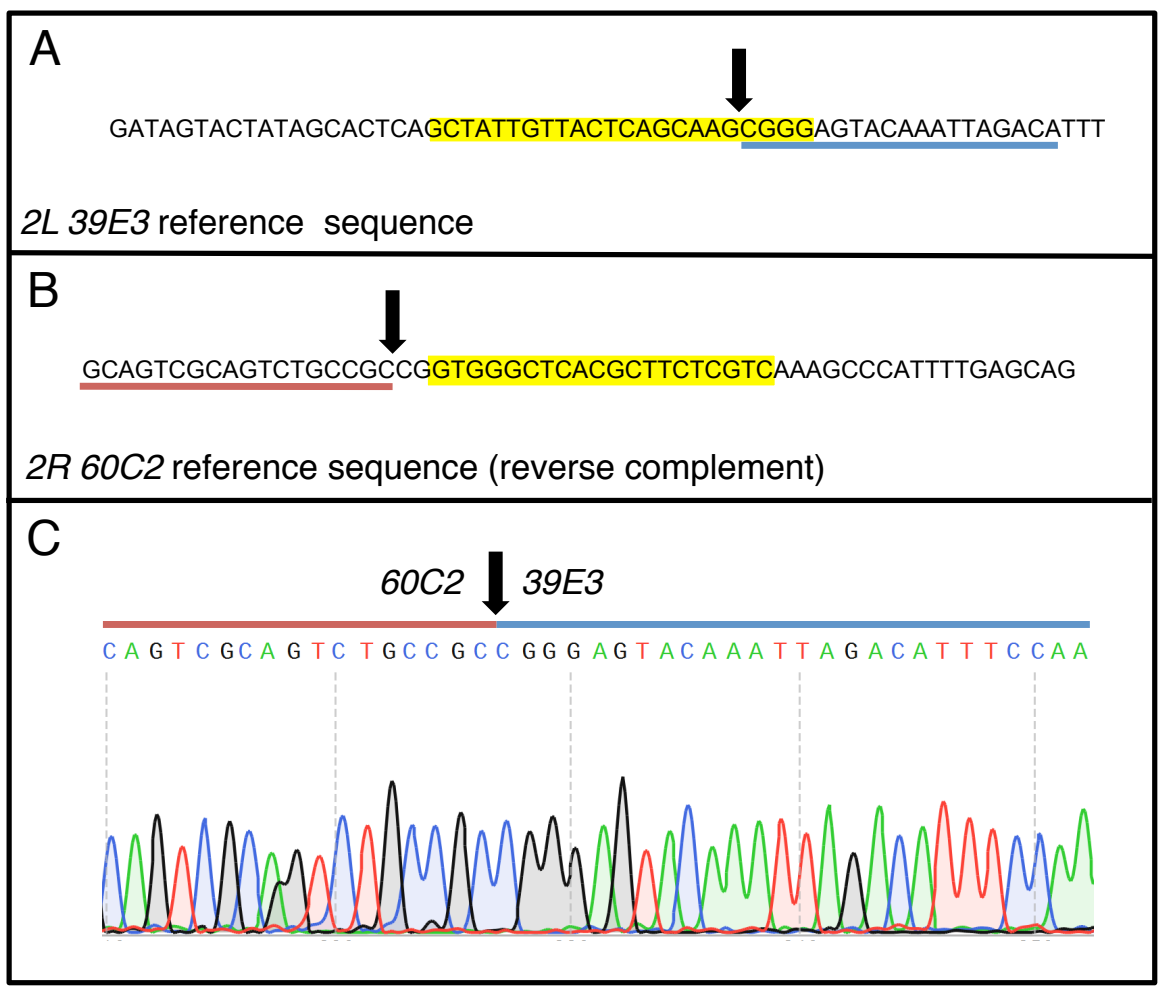

Figure 7 